\title{
Effectiveness of a psycho-educational intervention to prevent postpartum parental distress and enhance infant well- being: study protocol of a randomized controlled trial
}

\author{
Marjolein Missler ${ }^{1,2^{*}} \mathbb{D}$, Roseriet Beijers ${ }^{3}$, Jaap Denissen ${ }^{2}$ and Annemieke van Straten ${ }^{1}$
}

\begin{abstract}
Background: The first months after birth can be challenging for parents, leading to parental distress and decreased well-being. Parents with high levels of distress are found to respond less adequately and sensitively to their infant, which in turn affects infant well-being and health. The goal of this study is to examine the effectiveness of a psycho-educational intervention to prevent postpartum parental distress and enhance the quality of caregiving and infant well-being. In contrast to other interventions, this intervention will be (1) offered already before birth, (2) offered to all parents-to-be, regardless of their risk of postpartum distress, and (3) include fathers. The proposed study examines the effectiveness of this intervention on (1) parenting distress, (2) quality of caregiving, and (3) the infant's well-being.

Methods/design: In this randomized controlled trial, 128 pregnant women and their partners will be recruited through midwifery practices and general media. Women with a complicated pregnancy, current psychopathology, insufficient Dutch language proficiency and without Internet access will be excluded. Parents will be randomized to either the intervention or a waitlist control group. The intervention consists of a booklet and video (offered prenatally), a home visit at 34-36 weeks of pregnancy and a telephone call 4 weeks after birth. Information and practical tools are provided on (1) sensitive responding and making contact with the baby, (2) crying, (3) feeding, and (4) sleeping. Assessments will take place at baseline (26-34 weeks of pregnancy), during the home visit (34-36 weeks of pregnancy), and 2,6, and 10 weeks after birth. The control group will be offered the intervention after the end of the study. The primary outcome is maternal parenting stress. Secondary outcomes are: paternal parenting stress, parental well-being, quality of caregiving, and infant well-being and health.
\end{abstract}

Discussion: The goal of this study is to test the effects of a psycho-educational prenatal parenting intervention to prevent postpartum parental distress and to enhance well-being in both parents and infants. When the intervention appears effective it can be implemented broadly because of its low costs. It will make support available for a large number of parents and their children.

Trial registration: Netherlands National Trial Register, ID: NTR6065. Registered on 15 September 2016.

Keywords: Parents, Pregnant women, Stress, Early intervention, Prevention, Infant health

\footnotetext{
* Correspondence: m.a.missler@vu.nl

'Department of Clinical, Neuro and Developmental Psychology and

Amsterdam Public Health Research Institute, Vrije Universiteit Amsterdam,

Amsterdam, Netherlands

2Department of Developmental Psychology, Tilburg University, Tilburg,

Netherlands

Full list of author information is available at the end of the article
} 


\section{Background}

Having a baby is an intense experience that turns the world of new parents upside down. While this life event often brings joy and happiness, the first months can also be challenging for parents (van Scheppingen, Denissen, Chung, Tambs, and Bleidorn, in press [61]). Parents have to develop a range of new skills in taking care for their infant, which requires extra effort and energy, while at the same time they have to deal with a significant lack of sleep. It is not surprising, therefore, that roughly $14 \%$ of mothers and $10 \%$ of fathers experience moderate or severe levels of postpartum distress [43], mainly consisting of depressive symptoms (e.g., [41]). This study examines the effectiveness of a psycho-educational intervention to prevent postpartum parental distress and enhance infant well-being.

\section{Parental distress and sensitive responding to the infant's needs}

Parental distress (including, but not limited to, stress related to the parental role) has been related to decreased quality of caregiving. Both maternal $[11,24]$ as well as paternal depression [46] have been associated with a range of negative outcomes for the child's emotional, cognitive and behavioral development [21]. The supposed mechanism underlying this link is that depressed parents are less able to respond sensitively to their infant $[24,37]$. Sensitivity refers to the extent to which caregivers timeously and appropriately respond to the infant's needs and signals [2]. Caregiver sensitivity has been identified as one of the most robust predictors of the development of a secure attachment bond between parent and child [3, 20, 30, 55]. Moreover, sensitivity has also been associated with increased social competence, resilience, regulatory capacities, and lower stress levels later in life [23, 25, 50, 52].

Other important characteristics of caregiving behavior are the type of feeding and the infant sleeping location that parents have arranged. Current Dutch guidelines advise parents to exclusively breastfeed for 6 months, and to share their room with the infant for 6 months (infant sleeping in its own crib in the parents' room, but not in the parental bed) $[56,57]$. The World Health Organization (WHO) also recommends exclusive breastfeeding until the infant is 6 months of age [63]. Breastfeeding has been related to important health benefits for the child, including protection against of infections, overweight, and diabetes [28, 62]. Moreover, breastfeeding has been related to increased cognitive development [29]. Next to breastfeeding, there is international agreement on the benefits of room-sharing during the first 6 months of the infant's life. Room-sharing protects from sudden infant death syndrome (SIDS; [47]). Also, being close to the infant facilitates the process of breastfeeding at night $[4,5]$. There are indications that the proximity of the parent functions as a buffer against the infant's distress $[8,58]$. Despite these recommendations, it has been demonstrated that both breastfeeding and roomsharing are frequently discontinued in the first 2 months of the infant's life [56, 57].

\section{Interventions to reduce parental distress}

There are a number of interventions available that have been proven effective in reducing parental distress ([3, 26, 33, 35, 60]). These interventions mostly start during the postpartum period and are tailored at families from high-risk populations, such as families with low socioeconomic status (SES), parents of an infant born prematurely, foster parents, and parents from children at risk for developing autism spectrum disorder. Moreover, these previous interventions mostly focused on only the mother. This is remarkable because research has shown that the father can play an important role; for example, in supporting the mother with breastfeeding and prolonging the breastfeeding period $[38,44]$. Also, intervention studies aimed at treating maternal depression showed that inclusion of the father in treatment led to greater successes of the treatment [54]. Paternal support might protect against maternal stress and the development of postpartum depression, and mitigate the negative effects of depression on the mother-infant interaction [60]. Furthermore, it has also been found that fathers tend to decrease their involvement in childcare when they suffer from psychological adjustment difficulties around the transition to parenthood [31]. Thus, previous research has pointed to the importance of including fathers in the intervention as this might have positive effects on the well-being of both parents as well as on the quality of their caregiving.

To our knowledge, there is only one intervention to date that focused on parents from both low- and highrisk groups. This intervention consists of psychoeducation about infant sleep and crying patterns, in combination with a telephone consult and a parent group, and showed positive results in a randomized controlled trial [27]. However, the intervention did not include information on sensitivity or feeding, and it was offered postpartum. Furthermore, as only data of the primary caregiver was collected, almost only data from the mother was available as a result.

\section{The current study}

We developed an intervention which can be offered during pregnancy, to both mothers and fathers, regardless of their risk of postpartum distress. The intervention consists of psycho-education (booklet and video) as well as a prenatal home visit and a postpartum telephone call. 
We expect that the proposed intervention will reduce maternal parenting stress. Furthermore, we expect that the intervention will reduce paternal parenting stress and parental distress in general. Moreover, we expect that parental well-being will be enhanced. By psychoeducating parents during pregnancy, we expect parents to experience more self-efficacy and satisfaction in fulfilling their roles. In this way, parents should be better able to provide high-quality caregiving (including breastfeeding and co-sleeping), leading to enhanced infant well-being (less problems with sleeping, crying and feeding, and better well-being and health) (Fig. 1).

\section{Objectives}

The primary objective of this study is to determine the postpartum effect of the intervention on maternal parenting stress. Secondary outcomes are: paternal parenting stress, parental distress in general (anxiety and depression) and parental well-being (satisfaction with the parental role, and self-efficacy in caring for the infant); quality of caregiving (bonding, breastfeeding, and co-sleeping); and infant well-being and health (crying, feeding, sleeping, well-being, and health).

\section{Methods/design}

\section{Trial design}

The study is a randomized controlled trial with two parallel groups: an intervention and a waitlist control group. The waitlist group will receive the intervention after the last assessment (10 weeks after the birth of their infant, see Fig. 2). Please refer to Additional file 1 for the Standard Protocol Items: Recommendations for Interventional Trials (SPIRIT) checklist.

\section{Study setting and recruitment}

Women will be recruited either through midwifery practices or general media (newspaper ads and Internet banners). The midwives will give the parents-to-be a flyer, referring to the study's website. Alternatively, parents can contact us directly by responding to online advertisements in which a link to the website is provided. On the website, a registration form can be filled out. Upon registering, parents will receive the digital information brochure of the study and an informed consent form. Following the Declaration of Helsinki, it will be clearly indicated on the informed consent form that study participation is entirely voluntary and that participants can withdraw from participation at any time without any negative consequences for them or their child. If parents decide to take part in the study, they will be asked to return the informed consent form. Interested parents who are not eligible for inclusion will receive a message explaining the reason(s) for their exclusion.

\section{Inclusion and exclusion criteria}

Pregnant women will be included in the study when they are before the 34th week of pregnancy and do not have severe medical conditions due to pregnancy (i.e., gestational diabetes or pre-eclampsia). Furthermore, women need sufficient Dutch language proficiency to understand the information offered to them during the intervention (through a booklet and a video). Also, access to the Internet is required to be able to complete the online questionnaires. Women with current psychopathology (defined as current treatment for psychopathology or treatment in the 6 months before inclusion) will be excluded. The partners of the pregnant women will also be asked to participate. However, women without a partner, or women with a partner who does not want to participate, can also enter the study.

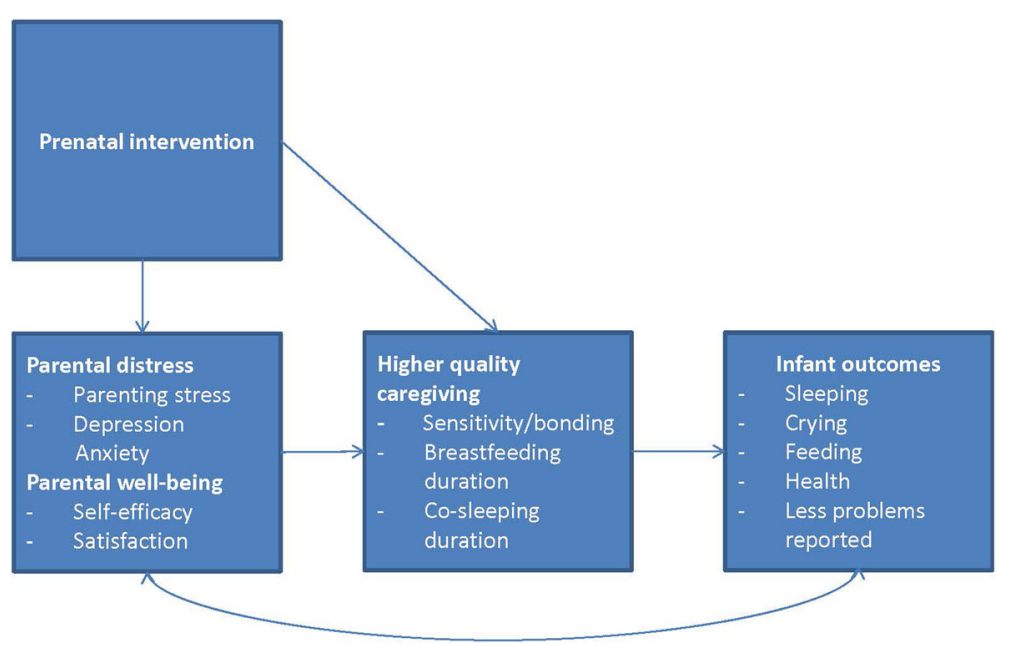

Fig. 1 Primary and secondary study outcomes (jpeg) 


\begin{tabular}{|c|c|c|c|c|c|c|c|}
\hline \multirow[b]{2}{*}{ TIMEPOINT } & \multirow{2}{*}{$\begin{array}{c}\text { Enrolment } \\
\begin{array}{c}1-34 \text { weeks } \\
\text { of } \\
\text { pregnancy } \\
(t-1)\end{array}\end{array}$} & \multirow{2}{*}{$\begin{array}{c}\text { Baseline (t0) } \\
\begin{array}{c}\text { 26-34 weeks of } \\
\text { pregnancy }\end{array} \\
(t 0) \\
\end{array}$} & \multirow{2}{*}{$\begin{array}{c}\begin{array}{c}\text { Prenatal } \\
\text { follow-up }\end{array} \\
\begin{array}{c}\text { 34-36 weeks } \\
\text { of }\end{array} \\
\begin{array}{c}\text { pregnancy } \\
\text { (t1) }\end{array} \\
\end{array}$} & \multicolumn{4}{|c|}{ Postnatal follow-up } \\
\hline & & & & $\begin{array}{c}2 \\
\text { weeks } \\
\text { after } \\
\text { birth } \\
(\mathrm{t} 2) \\
\end{array}$ & $\begin{array}{c}4 \\
\text { weeks } \\
\text { after } \\
\text { birth }\end{array}$ & $\begin{array}{c}6 \\
\text { weeks } \\
\text { after } \\
\text { birth } \\
(\mathrm{t} 3) \\
\end{array}$ & $\begin{array}{c}10 \\
\text { weeks } \\
\text { after } \\
\text { birth } \\
(\mathrm{t} 4) \\
\end{array}$ \\
\hline \multicolumn{8}{|l|}{ ENROLMENT: } \\
\hline Eligibility screen & & $\mathrm{X}$ & & & & & \\
\hline Informed consent & $\mathrm{x}$ & & & & & & \\
\hline Randomization & & $\mathrm{x}$ & & & & & \\
\hline \multicolumn{8}{|l|}{ INTERVENTIONS: } \\
\hline Intervention & & & $\mathrm{X}$ & & $\mathrm{X}$ & & \\
\hline \multicolumn{8}{|l|}{ Control } \\
\hline \multicolumn{8}{|l|}{ ASSESSMENTS: } \\
\hline Demographics & & $\mathrm{X}$ & & & & & \\
\hline Attachment style & & $\mathrm{x}$ & & & & & \\
\hline Marital satisfaction & & $\mathrm{X}$ & & & & & $\mathrm{X}$ \\
\hline $\begin{array}{l}\text { Parenting distress } \\
\text { Parental well-being }\end{array}$ & & $\mathrm{X}$ & $\mathrm{X}$ & & & $\mathrm{X}$ & $\mathrm{X}$ \\
\hline Quality of caregiving & & & & & & $\mathrm{X}$ & $\mathrm{X}$ \\
\hline Infant well-being & & & & & & $\mathrm{X}$ & $\mathrm{X}$ \\
\hline $\begin{array}{c}\text { Delivery } \\
\text { characteristics }\end{array}$ & & & & $\mathrm{X}$ & & & \\
\hline
\end{tabular}

Fig. 2 Standard Protocol Items: Recommendations for Interventional Trials (SPIRIT) Figure with trial design and outcome assessments. After screening for eligibility, participants are asked to sign an informed consent form and to complete the baseline questionnaire. Then, participants are randomized in either the intervention or the control group. The intervention starts with a home visit (at t 1 ) and a postnatal telephone call (4 weeks after birth). Measurements take place at 26-34 weeks of pregnancy (baseline; t0); 34-36 weeks of pregnancy (t1); 2 weeks after birth (t2; delivery characteristics); 6 weeks after birth (t3); and 10 weeks after birth (t4)

\section{Sample size calculation}

We expect an effect size of 0.50 (Cohen's $d$ ). This is based on the work of Hiscock et al. [27]. Their intervention, aimed at preventing infant sleep and crying problems, showed an odds ratio of 0.57 (converted into a Cohen's $d$ of 0.48 ) on symptoms of maternal depression in favor of the intervention group. Using a power of $80 \%$, an alpha of 0.05 , we need to include 64 participants in both conditions, 128 in total, to answer our primary research question.

\section{Randomization}

An independent researcher will generate random number sequences using random allocation software with a 1:1 ratio. We will use random sequence blocks (blocks between 6 and 8), stratified by birth order (first child/ no first child) and paternal participation (yes/no). An independent researcher will allocate each consenting participant to either the control or the intervention group. Given the nature and design of the study, blinding after randomization is not possible. Participants will receive the outcome of randomization by e-mail. Please refer to Fig. 3 for an overview of participant's flow through the study.

\section{Intervention}

Parents will receive access to the psycho-education materials (booklet and video) directly after randomisation, which is between the 26th and the 34th weeks of pregnancy (depending on when the parents apply for participation). The intervention further consists of two support sessions: one home visit between 34 and 36 weeks of pregnancy and one telephone call 4 weeks after birth. 


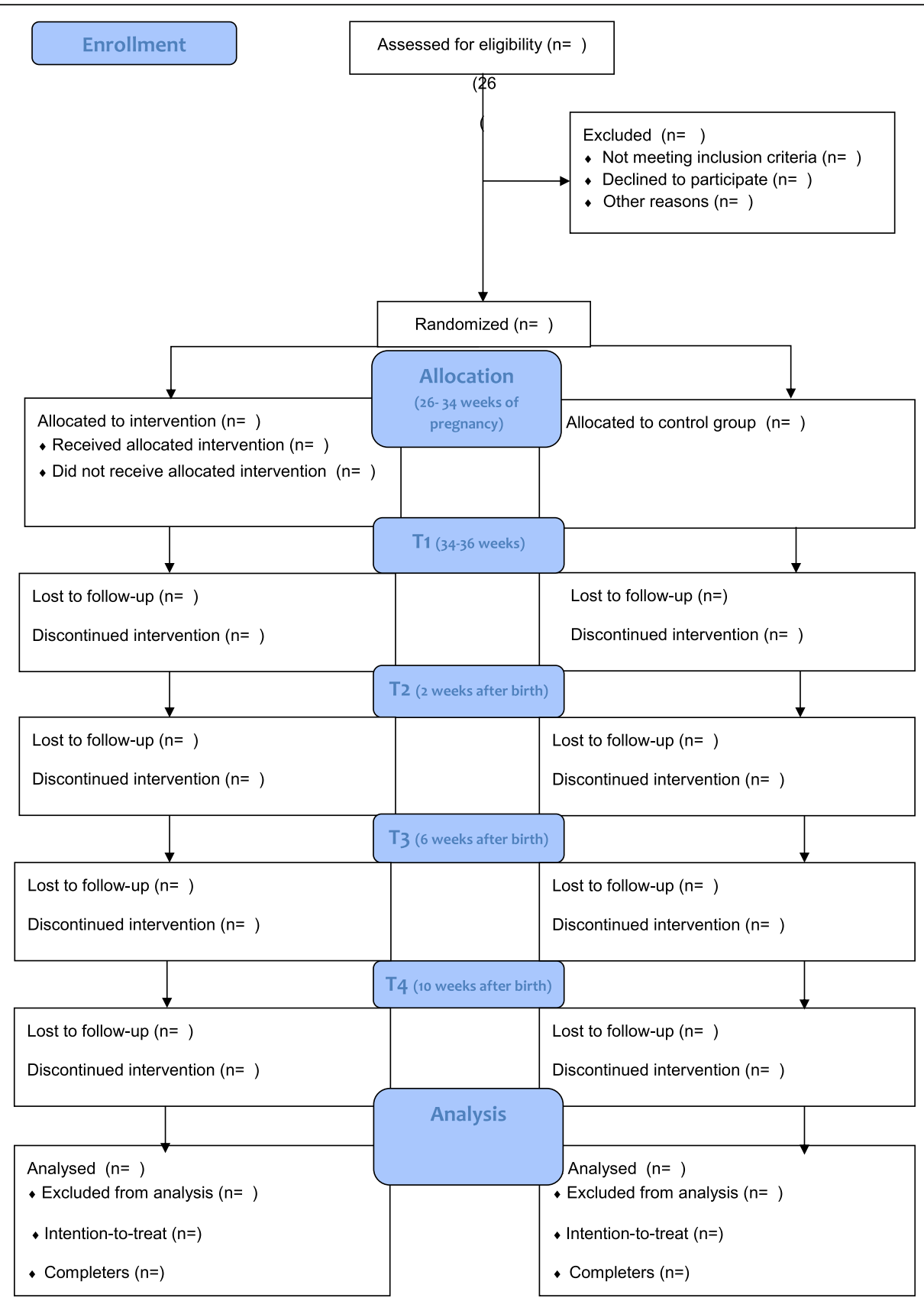

Fig. 3 Consolidated Standards of Reporting Trials (CONSORT) flow diagram. After screening for eligibility, participants are randomized in either the intervention or the control group. Follow-up measurements take place at 34-36 weeks of pregnancy ( $\mathrm{t} 1$ ); 2 weeks after birth (t2; delivery characteristics); 6 weeks after birth (t3); and 10 weeks after birth (t4)

\section{Psycho-educational booklet and video}

In general, the intervention conveys the importance of responding sensitively to the needs of the infant. The psycho-educational booklet developed by Hiscock et al. [27] was used as a starting point. We developed and extended the intervention further based on recent empirical research and together with a number of different experts on attachment (researchers), breastfeeding (lactation consultants), and pre and postnatal care for both mother and child (midwives). Our booklet consists of four chapters about: (1) sensitive responding and making contact with the baby, (2) crying, (3) feeding, and (4) sleeping. The first chapter includes information about contact-seeking behavior of the baby and how to interpret infant's signals of distress and respond to these adequately (e.g., [9]). The chapter also includes information explaining the needs of the infant (such as the need for closeness, skin-to-skin contact, and 
sensitive care), followed by a discussion about the parent's own needs and how to attend to these. Then, the booklet continues with a chapter about crying. The normal crying curve is addressed, and different tools are provided on how to sooth a baby. Also, potential causes of excessive crying are discussed. The third chapter about feeding includes information about hunger signals that the baby might convey. Information is given on breastfeeding, and its positive effects for both mother and child. Practical information about the use of formula milk is also provided. The fourth and final chapter on sleeping provides information on average sleeping patterns and sleep signals that a tired baby might convey. Moreover, the importance of room-sharing during the first 6 months will be discussed and suggestions on when and how to support the infant in learning to sleep alone in a separate room are given. It is also important to note here that sensitive care is put central in our intervention, and that, for example, "crying it out" and "camping it out" techniques [32], in which parents are instructed to let infants cry for a specified amount of time, are not included in our intervention. The booklets ends with a section about common baby myths, such as "Keeping the baby awake all day, will make the baby sleep better during the night" or "When I pick up my baby every time, I will spoil the baby." The booklet consists of written text and pictures. Most importantly, the booklet provides many examples on how to use the information in daily life. In the booklet, it is explained that the provided information is not meant to be prescriptive; rather, it encourages parents to distil those elements that are most helpful for them in their specific situation (while keeping the principles of sensitive care in their mind). This way, parents can start discovering which tools fit best with their and their infant's preferences and needs.

At the same time as the booklet, participants receive access to an online video. This video is developed by psychologists who are experts in promoting the mental health of babies and their parents by translating academic knowledge into easily accessible interventions (Stichting Babywerk, The Netherlands). The video serves as an illustration of the topics which are described in the booklet and shows practical examples. It is a story format in which the experiences of (upcoming) parents are shown. An expert on infant development comments on each of the fragments. Also, after each fragment, participants are confronted with a thought-provoking question about what they just have seen. The aim of these questions is to stimulate participants to think about the information in relation to their lives. For example, in the story, the upcoming mother is worried that she will be unable to put her mobile phone away when her baby is awake. At this point, the participant will be asked what their thoughts are about this issue. Next, the expert explains why being able to recognize and respond to the infant's signals is important. After the explanation, the video shows how to notice and respond to an infant seeking eye contact. Watching the video and responding to the questions takes about 15-20 min. The parents are encouraged to put this advice into practice after the birth of their child.

\section{Support sessions}

Parents will be visited at home during weeks $34-36$ of the pregnancy. The primary aim of this home visit is to discuss the provided information (booklet and video) and to answer any questions about the materials the parents might have. The secondary aim is to explain that the provided information is not meant to be prescriptive; rather, we aim to provide parents with a set of tools (the practical examples) that they can choose from. By means of provided materials, we ask participants to start discovering which tools fit best with their and their infant's preferences and needs.

Four weeks after the birth of their child, parents will be contacted by telephone. During this call, we will ask the parents how they and their child are doing, and whether they are experiencing any problems in implementing the information from the booklet and the video in their daily lives. Also, parents are given the opportunity to ask questions with regard to their infant's feeding, crying, sleeping, and contact-seeking behavior. They can also ask questions with regard to their own well-being (i.e., experienced stress or feelings of anxiety). Both the home visit as well as the telephone call will be performed by the corresponding author, who has a background in clinical psychology and infant development.

\section{Waitlist control group and care-as-usual}

Parents in the waitlist control group will receive the same materials as the parents in the intervention group after the last assessment (10 weeks after birth). The information can still be of use for them at that time. Both groups, the intervention and control group, will have access to care-as-usual during the postpartum period. In the Netherlands, this consists of regular consults with a specialized nurse at home (2 weeks after birth) or at the well-baby clinic (at 4, 8, and 12 weeks after birth) during which the health of the infant is checked upon and the infant's growth is followed.

\section{Assessments and outcomes}

We will measure at baseline (26-34 weeks of pregnancy, t0); 34-36 weeks of pregnancy (t1); 2 weeks after birth (t2); 6 weeks after birth (t3); and 10 weeks after birth (t4). All assessments will take place online, except for the completion of the infant behavior diary (t3; Fig. 2). 


\section{Baseline variables}

At baseline ( $\mathrm{t} 0$ ), we assess the demographic characteristics of the parents (date of birth; marital status, income, educational level, and working hours).

Furthermore, to control for possible insecure attachment styles of the parents (which can have an impact on their caregiving quality (e.g., [22]), we will measure attachment styles of the parents using the short form of the Experiences in Close Relationships Questionnaire (ECR-short form; $\left.[15,34]^{1}\right)$. The 12 items of this instrument are derived from the avoidance and anxious attachment subscales of the ECR-R (six items of each subscale; [10]). Response options vary from 1 (strongly disagree) to 7 (strongly agree). The avoidance subscale measures the need to stay independent from others and to avoid intimacy ([34], see also [7]). The anxiety subscale is concerned with the degree to which the subject worries about rejection and abandonment $([7,34])$. Following recoding of items $15,25,27,29$, and 31 ; for each subscale (anxiety and avoidance) an average score of between 1 and 7 can be computed. Higher scores reflect more attachment anxiety and avoidance. The ECR-short form showed good psychometric properties in different samples: Lafontaine et al. [34] reported Cronbach's alpha's of .78 to .87 for the anxiety subscale and .74 to .83 for the avoidance subscale.

\section{Primary outcome}

The primary outcome is maternal parenting stress (assessed at $\mathrm{t} 0 ; \mathrm{t} 1 ; \mathrm{t} 3$; and $\mathrm{t} 4$ ) and is measured with 10 items of the Dutch version of the Parenting Stress Index (PSI; $[1,18])$. The complete version of this instrument consists of 123 items. A shortened version of 25 items is available [19]. However, since the PSI has been originally developed for parents of children up to 14 years [18], our aim was to select the 10 items that are most relevant for parents of a newborn child. Following the procedure of Missler et al., [39], we selected those items that, in our view, best captured their experience. One item was added because it was considered to be central to the parenting stress construct (at least for parents with very young children): "The responsibility I have for my children weighs on me" Missler et al., [39]. An example item is: "I feel restricted by my responsibilities as a parent." For the administration before birth ( $\mathrm{t} 0$ and $\mathrm{t} 1$ ), the items were slightly rephrased to capture possible stress-raising expectations of parenthood: "I expect to feel restricted by my responsibilities as a parent." Response options vary from 1 (totally disagree) to 6 (totally agree). A total score can be derived by summing the individual item scores. This total score (including the added item) ranges from 11 (no stress) to 66 (very high stress).

\section{Secondary outcomes}

Secondary outcomes are paternal parenting stress, parental well-being, quality of caregiving, and infant wellbeing and health.

Paternal parenting stress Paternal parenting stress will be measured with the same 10 items of the Dutch version of the Parenting Stress Index (PSI; $[1,18]$ ) used for measuring maternal parenting stress. For more details, see above.

Parental well-being Parental well-being is defined as: (1) depressive symptoms, (2) symptoms of anxiety, (3) satisfaction with the parental role, (4) parental selfefficacy, and (5) sleep quality and quantity.

1. Depressive symptoms will be measured with the Edinburgh Postnatal Depression Scale (EPDS; [17]; Dutch translation Pop, Komproe, and Van Son, 1992). This scale consists of 10 items. Participants can indicate the experienced frequency of each depression-related statement on a 4-point scale. Items are scored $0,1,2$, or 3 (items 3, 5, 6, 7,8, 9, and 10 are reverse scored). Total scores range from 0 (no depressed feelings) to 30 (severely depressed feelings). The scale shows good psychometric properties: Pop et al. [45] reported an internal consistency of .82 (Cronbach's alpha) and sufficient concurrent validity

2. Symptoms of anxiety will be measured with the seven items of the anxiety subscale of the Hospital Anxiety and Depression Scale (HADS; [42, 51]). For each item, participants can indicate on a 4-point scale how much anxiety they experience. Total scores on the anxiety subscale vary from 0 (no anxiety) to 21 (severe anxiety). Spinhoven et al. [51] reported good psychometric properties for the Dutch version

3. Satisfaction with the parental role will be measured with three items of the Dutch translation of the Parenting Stress Index (PSI; [18, 19, 36]). Following the procedure of Missler et al., [39], four items were added to this scale. Adding items was necessary because to our knowledge, no measure of parenting satisfaction currently exists. We thus decided to use this composed scale. An example item is: "I enjoy spending time with my child." For the administration before birth ( $\mathrm{t} 0$ and $\mathrm{t} 1$ ), the items were rephrased such that the expectation of parents of their satisfaction with their new role after the birth of their child became central: "I expect I will enjoy spending time with my child"

4. Parents will be asked to rate their efficacy as a parent on a 5-point scale, ranging from 1 (not very good) to 5 (a very good parent; [16, 27, 49]) 
5. Sleep quality and quantity is measured with two items of the Pittsburgh Sleep Quality Index (PSQI; [12]) adapted by [16]. The quality item is: "Over the last 2 weeks, how would you rate your own sleep quality?" Response options are: "Not nearly good enough"; "Not quite good enough"; "Good enough"; and "More than good enough." The quantity item is: "Over the last 2 weeks, how would you rate your own sleep quantity?", with the following response options: "Not nearly enough"; "Not quite enough"; "Enough"; and "More than enough."

Quality of caregiving Quality of caregiving will be measured by assessing the bonding between parent and child, the duration of breastfeeding, and the duration of room-sharing at 10 weeks postpartum.

1. Bonding will be measured with the Maternal Postnatal Attachment Scale (MPAS; [14, 59]). This scale consists of 19 statements referring to parentchild relationship. Each statement can be answered on a 2-point; 4-point; or 5-point scale. An example item is: "When I am with the baby, I feel tense and fearful." Total scores range from 19 to 95 and can be computed by summing up individual item scores. Low total scores indicate bonding problems between parent and child. Van Bussel et al. [59] reported a Cronbach's alpha of .75 of the MPAS when administered 8-12 weeks after birth

2. Breastfeeding will be assessed by measuring the duration (in weeks) of breastfeeding and/or breastfeeding mixed with bottle-feeding

3. Room-sharing will be measured as the duration (in weeks) of room-sharing (the infant sleeps in the room of the parents at night)

\section{Infant well-being and health}

The well-being of the infant will be measured through a diary (72 h at 6 weeks postpartum) assessing crying, sleeping, and feeding [6]. Parents are supposed to rate precision in 5-min slots using different symbols. The following behaviors will be assessed: asleep, awake, and being content, awake and being fussy, awake and crying, awake and feeding; awake and sucking (thumb/dummy). Parents can also indicate the time and duration of feeding, and the type of feeding (breastmilk (through breastfeeding or bottle) or formula milk). Parents are asked whether the rated $72 \mathrm{~h}$ period can be viewed as a "typical" period or not. Furthermore, we will ask parents at 6 and 10 weeks after birth whether they are experiencing a problem with infant sleep (day or night), crying or feeding, and if so, to rate the severity of this problem on a 7point Likert scale ranging from 1 (hardly any problem) to 7 (a severe problem) [27]. Infant health is measured in terms of somatic indices and somatic problems (at 6 and 10 weeks after birth). Parents are asked about their infant's weight, length, and head circumference and will also be asked to indicate whether their child has experienced fever, a runny nose, any coughing, ocular inflammation, or diaper rash. Additionally, parents are asked if their child had experienced some other medical condition and whether their child has been using any medication.

\section{Birth}

Variables related to the delivery and the birth are assessed 2 weeks after birth: birth weight, Apgar score, spontaneous delivery, caesarean section, birth complications. We will test whether there are differences between the intervention and control groups for these variables (measuring possible stress factors during the delivery) in the analyses.

\section{Marital satisfaction}

Marital satisfaction is measured with the global satisfaction items of The Investment Model Scale (IMS; [48]; Dutch translation: Montgomery, Peeters, Schaufeli, and Panagopoulou, [40]). This scale consists of five items, with answering options varying from 1 (totally disagree) to 9 (totally agree). An example item is: "My relationships fulfills my needs for intimacy." The total score ranges from 5 (not satisfied) to 45 (very satisfied). Montgomery et al., 1998, report a Cronbach's alpha of .93 . Again, we will test for potential differences on this variable between the two groups.

\section{Intervention uptake}

Finally, we measure the uptake of the intervention at 10 weeks after birth by asking parents whether they have read and watched the materials before the birth of their child. We also ask them whether they looked into the materials again after the birth of their child. Additionally, we monitor online the duration and frequency of watching (parts) of the video. Furthermore, we ask them to rate the frequency of using the information in their daily lives, with the item: "How many times did you use the information from the booklet, video, or the home visit during the daily care for your baby?" Response options were: "Daily"; "Several times a week"; "About once a week"; "About once every 2 weeks"; "About once a month"; "Never." We also ask them to rate the usefulness of the booklet, video, and the home visit on a 5-point scale ranging from "Not very useful" to "Very useful."

\section{Data management}

We will keep one file in which research identifiers are linked to participant's names and (e-mail) addresses. This file will be encrypted and password protected and 
only be available to the main researchers. All on- and offline data will be safely stored at the university using research identifier only.

\section{Statistical analysis}

The study will be conducted in adherence to the Consolidated Standards of Reporting Trials (CONSORT) Statement. We will perform intention-to-treat analysis, in which all patients who are randomized will be analyzed (independent of treatment or study completion), as well as completers-only analyses. Completers are defined as participants who completed all measurements. Baseline data will be explored to see if there are differences between the group that remains in the study and the group that drops out.

\section{Primary outcome}

We will compare the parenting stress scores between the two groups using a mixed multilevel model. This way, we can account for the nested structure of the data (mothers and fathers that belong to the same couple). Including both mothers and fathers in one analysis, will positively affect the power of the analyses. Furthermore, the analysis is also robust for missing data (Tabachnik and Fidell, [53]) and unequal sample sizes (more participating mothers than fathers). We will test interaction terms between parental sex (mother versus father) and intervention group (intervention versus control), to test whether the (possible) effect of the intervention is the same for both mothers and fathers. We will also calculate the between-group effect sizes at 6 (t3) and 10 weeks postpartum (t4). We will calculate Cohen's $d$ by subtracting the two mean scores and dividing them by the pooled standard deviations. A Cohen's $d$ of 0.2 can be assumed small, 0.5 to be moderate, and 0.8 to be large [13].

\section{Secondary outcomes}

We will repeat the mixed multilevel models, and the calculation of Cohens' $d$, for all outcomes of parental wellbeing. Next, to determine the effect of the intervention on the quality of caregiving after birth, we will add higher-quality caregiving as a secondary outcome variable to the model. Furthermore, to test whether prenatal well-being of the parents influences postpartum caregiving quality, we will use parental well-being (measured at 26 and 34 weeks of pregnancy) as a predictor of higherquality caregiving. Finally, we will determine the effect of prenatal parental well-being on infant outcomes postpartum (mediated by higher-quality caregiving). For each of the outcomes we will calculate the between-group effect size at $\mathrm{t} 3$ and $\mathrm{t} 4$ and their $95 \%$ confidence interval. Gender will be added as an interaction term to the model, to see if there are differences between mothers and fathers.

Demographic data, attachment style of the parents and data related to the delivery and birth (birth weight, Apgar score, spontaneous delivery, caesarean section, birth complications) will be used as control variables in the model.

\section{Data monitoring}

Since the current intervention is a non-pharmacological intervention, it is unlikely that adverse effects due to the intervention will occur. Therefore, installing a Data Monitoring Safety Board does not seem warranted.

\section{Harms}

We consider this study to have negligible risks. One possibility is that the parents become aware of possible postpartum stressors and, therefore, become more stressed instead of less stressed. To prevent this rise of stress levels in both groups, we will take specific action. We will underline to the parents from the intervention group that we do not expect them to implement the intervention perfectly. We will explicitly give them the opportunity to make their own choices regarding the advice given in the booklet. Parents are completely free to determine which of the tools provided in the intervention they would like to use.

For the control group, we will stress that the intervention is a way of extra support, not the solution to prevent problems. They will be granted access 10 weeks after birth.

\section{Amendments}

All substantial amendments will be notified to the Medical Ethics Committee (METC) and to the competent authority.

Non-substantial amendments will not be notified to the accredited METC and the competent authority, but will be recorded and filed by the sponsor.

\section{Dissemination policy}

MM and RB will process the data, and both positive and negative findings will be disclosed, unreservedly. Results will be submitted for publication to peer-reviewed scientific journals. The participating parents as well as the participating midwifery centers will receive updates on the study's progress. At the end of the project, results and conclusions will be presented to all those involved.

\section{Discussion}

This goal of this study is to examine the effectiveness of a psycho-educational intervention to prevent postpartum parental distress and enhance infant well-being. In contrast to other interventions, this intervention will be 
offered (1) already before birth, (2) to all parents-to-be, regardless of their risk of postpartum distress, and (3) to include fathers.

We expect parents to experience less distress and higher well-being after the birth of their child compared to parents who do not receive this intervention. In turn, we expect that this will have positive effects on the quality of their caregiving, and ultimately on the infant's well-being and health, resulting in less parent-reported problems with sleeping, crying, and feeding, and increased infant health.

The intervention is focused on sensitive caregiving; that is, responding timely and adequately to the infant's needs. The booklet consists of four chapters; on making contact and sensitive responding, crying, feeding, and sleeping. A video is available to illustrate the topics mentioned in the booklet. During a prenatal home visit, the provided materials will be discussed. After birth, parents receive a supportive telephone call. Throughout the intervention, it will be underlined that no part of the intervention is meant to be prescriptive; rather, the intervention has been developed to support parents in choosing those elements that are most helpful for them in their specific situation. Indeed, by offering the intervention we aim to stimulate parents to think about and discuss the information provided, whether or not they will implement everything that they have learned. This way, we expect both parents to be better equipped for the transition to parenthood.

In our view the timing of implementation of our psycho-educational intervention is crucial. By intervening already during pregnancy (and continuing to offer support during the first months after birth), our aim is to prevent or mitigate distress among parents and infants - and related problems with breastfeeding and cosleeping - during the first hours, days, and weeks of the infant's life. In this way, the infant's health and early development can optimally benefit. The intervention is low-cost, easy to implement, and can be distributed on a large scale: a large number of parents and infants can thus be supported during a crucial period of their lives.

\section{Trial status}

Recruitment started in November 2016 and is ongoing. In November 2016, the first participants enrolled. Currently, 59 participants have signed the informed consent form and are thus participating in the study (nine of them have completed the study protocol).

\section{Endnotes}

${ }^{1}$ Items of the ECR-short form are extracted from the ECR-R [10], and this version has been validated in Dutch [15].

\section{Additional file}

Additional file 1: Standard Protocol Items: Recommendations for Interventional Trials (SPIRIT) checklist. (DOC $122 \mathrm{~kb}$ )

\section{Abbreviations}

ECR: Experiences in Close Relationships Questionnaire; EPDS: Edinburgh Postnatal Depression Scale; HADS: Hospital Anxiety and Depression Scale; IMS: The Investment Model Scale; MPAS: Maternal Postnatal Attachment Scale; PSI: Parenting Stress Index; PSQI: Pittsburgh Sleep Quality Index; SES: Socioeconomic status; SIDS: Sudden infant death syndrome; WHO: World Health Organization

\section{Acknowledgements}

Not applicable.

\section{Funding}

This research is funded by the Netherlands Organization for Scientific Research (NWO: 406.14.106)

\section{Availability of data and materials \\ Not applicable}

\section{Authors' contributions}

MM participates in the study design, participant recruitment, trial coordination, and drafted the manuscript. RB participates in the study design and supervised writing the manuscript. JD is initiator of the study, obtained funding and provided feedback on the draft of this paper. AvS is the principle investigator, participated in the study design and supervised writing the manuscript. All authors have read and approved the final manuscript.

\section{Ethics approval and consent to participate}

Ethical approval has been obtained from the Medical Ethical Committee Brabant (NL58528.028.16/P1620). We will obtain informed consent from all participants in the study.

\section{Competing interests}

The authors declare that they have no competing interests.

\section{Publisher's Note}

Springer Nature remains neutral with regard to jurisdictional claims in published maps and institutional affiliations.

\section{Author details}

${ }^{1}$ Department of Clinical, Neuro and Developmental Psychology and Amsterdam Public Health Research Institute, Vrije Universiteit Amsterdam, Amsterdam, Netherlands. ${ }^{2}$ Department of Developmental Psychology, Tilburg University, Tilburg, Netherlands. ${ }^{3}$ Behavioural Science Institute, Radboud University, Nijmegen, Netherlands.

Received: 12 July 2017 Accepted: 24 November 2017 Published online: 04 January 2018

\section{References}

1. Abidin R. Parenting Stress Index: manual. Charlottesville: Pediatric Psychology Press; 1983.

2. Ainsworth MS, Blehar MC, Waters E, Wall S. Patterns of attachment: a psychological study of the strange situation. 3rd ed. Oxford: Lawrence Erlbaum; 1978.

3. Bakermans-Kranenburg MJ, Van IJzendoorn MH, Juffer F. Less is more: metaanalyses of sensitivity and attachment interventions in early childhood. Psych Bull. 2003;129:195-215.

4. Ball HL. Breastfeeding, bed-sharing, and infant sleep. Birth. 2003;30:181-8.

5. Ball HL, Ward-Platt MP, Heslop E, Leech SJ, Brown KA. Randomised trial of infant sleep location on the postnatal ward. Arch Dis Child. 2006;91:1005-10.

6. Barr RG, Kramer MS, Boisjoly C, McVey-White L, Pless IB. Parental diary of infant cry and fuss behavior. Arch Dis Child. 1988;63:380-7.

7. Bartholomew K, Horowitz LM. Attachment styles among young adults: a test of a four-category model. J Pers Soc. 1991;61:226-44. 
8. Beijers RJ, Riksen-Walraven M, De Weerth C. Cortisol regulation in 12month-old human infants: associations with the infant's early history of breastfeeding and co-sleeping. Stress. 2013;16:267-77.

9. Beijers R, Cillessen L, Zijlmans M. An experimental study on mother-infant skin-to-skin contact in full-terms. Inf Beh Dev. 2016;43:58-65.

10. Brennan KA, Clark C, Shaver PR. Self-report measurement of adult attachment: an integrative overview. In: Simpson JA, Rholes WS, editors. Attachment theory and close relationships. New York: Guilford Press; 1998. p. 46-76.

11. Brennan PA, Hammen C, Andersen MJ, Bor W, Najman JM, Williams GM. Chronicity, severity, and timing of maternal depressive symptoms: relationships with child outcomes at age 5. Dev Psych. 2000;36:759-66.

12. Buysse DJ, Reynolds III CF, Monk TH, Berman SR, Kupfer DJ. The Pittsburgh Sleep Quality Index: a new instrument for psychiatric practice and research. Psych Res. 1989;28:193-213.

13. Cohen J. A power primer. Psych Bull. 1992;112:155-9.

14. Condon JT, Corkindale CJ. The assessment of parent-to-infant attachment: development of a self-report questionnaire. J Rep Inf Psych. 1998;16:57-76.

15. Conradi HJ, Gerlsma C, Van Duijn M, De Jonge P. Internal and external validity of the experiences in close relationships questionnaire in an American and two Dutch samples. Eur J Psych. 2006;20:258-69.

16. Cook F, Bayer J, Le HND, Mensah F, Cann W, Hiscock H. Baby business: a randomized controlled trial of a universal parenting program that aims to prevent early infant sleep and cry problems and associated parental depression. BMC Ped. 2012;12:13.

17. Cox JL, Holden JM, Sagovsky R. Detection of postnatal depression: development of the 10-item Edinburgh Postnatal Depression Scale. Brit J Psych. 1987; 150:782-8.

18. De Brock AJLL, Vermulst AA, Gerris JRM, Abidin RR. Nijmeegse ouderlijke stress index: meetinstrument voor de vaststelling van stress bij opvoeders. Lisse, The Netherlands: Swets \& Zeitlinger; 1992.

19. De Brock AJLL, Vermulst AA, Gerris JRM, Abidin RR. NOSIK. Lisse, The Netherlands: Swets \& Zeitlinger; 1992.

20. De Wolff MS, Van IJzendoorn MH. Sensitivity and attachment: a meta-analysis on parental antecedents of infant attachment. Soc Res Child Dev. 1997;68:571-91.

21. Downey G, Coyne JC. Children of depressed parents: an integrative review. Psych Bull. 1990;108:50-76.

22. Edelstein RS, Weede Alexander K, Shaver PR, Schaaf JM, Quas JA, Lovas GS, Goodman GS. Adult attachment style and parental responsiveness during a stressful event. Attach Hum Dev. 2004;6:31-52.

23. Fearon RP, Bakermans-Kranenburg MJ, Van IJzendoorn MH, Lapsley AM, Roisman Gl. The significance of insecure attachment and disorganization in the development of children's externalizing behavior: a meta-analytic study. Child Dev. 2010;81:435-56

24. Gelfand DM, Teti DM. The effects of maternal depression on children. Clin Psych Rev. 1990;10:329-53.

25. Groh AM, Roisman GI, Van IJzendoorn MH, Bakermans-Kranenburg MJ, Fearon RP. The significance of insecure and disorganized attachment for children's internalizing symptoms: a meta-analytic study. Child Dev. 2012;83:591-610.

26. Guttentag CL, Landry SH, Williams JM, Baggett KM, Noria CW, Borkowski JG, Swank PR, Farris JR, Crawford A, Lanzi RG, Carta JJ, Warren SF, Ramey SL. "My baby and me": effects of an early, comprehensive parenting intervention on atrisk mothers and their children. Dev Psych. 2014;50:1482-96.

27. Hiscock HA, Cook F, Bayer J, Le H, Mensah F, Cann W, Symon B, St. JamesRoberts I. Preventing early infant sleep and crying problems and postnatal depression: a randomized trial. Ped. 2014;133:346-54

28. Horta BL, Victora CG. Long-term effects of breastfeeding: a systematic review. Geneva: World Health Organization; 2013.

29. Horta BL, Loret de Mola C, Victora CG. Breastfeeding and intelligence: a systematic review and meta-analysis. Act Ped. 2015;104:14-9.

30. Isabella RA, Belsky J, Von Eye A. Origins of infant-mother attachment: an examination of interactional synchrony during the infant's first year. Dev Psych. 1989;25:12-21.

31. Jia R, Kotila LE, Schoppe-Sullivan SJ, Kamp Dush CM. New parent's psychological adjustment and trajectories of early parental involvement. J Mar Fam. 2016;78:197-211.

32. Kempler L, Sharpe L, Miller CB, Bartlett DJ. Do psychosocial sleep interventions improve infant sleep or maternal mood in the postnatal period? A systematic review and meta-analysis of randomised controlled trials. Sleep Med Rev. 2016;29:15-22.

33. Klein Velderman M, Bakermans-Kranenburg MJ, Juffer F, Van IJzendoorn MH. Effects of attachment-based interventions on maternal sensitivity and infant attachment: differential susceptibility of highly reactive infants. J Fam Psych. 2006;20:266-74

34. Lafontaine MF, Brassard A, Lussier $Y$, Valois P, Shaver PR, Johnson SM. Selecting the best items for a short-form of the Experiences in Close Relationships questionnaire. Eur J Psych Assess. 2006;32:140-54.

35. Landry SH, Smith KE, Swank PR. Responsive parenting: Establishing early foundations for social, communication, and independent problem solving skills. Dev Psych. 2006;42:627-642.

36. Loyd BH, Abidin RR. Revision of the Parenting Stress Index. J Ped Psych. 1985;10:169-77.

37. Lovejoy MC, Graczyk PA, O'Hare E, Neuman G. Maternal depression and parenting: a meta-analytic review. Clin Psych Rev. 2000;20:561-92.

38. Maycock B, Binns CW, Dhaliwal S, Tohotoa J, Hauck Y, Burns S, Howat P. Education and support for fathers improves breastfeeding rates: a randomized controlled trial. J Hum Lac. 2013;29:484-90.

39. Missler MA, Stroebe M, Van der Laan G. The work-home interface: The role of home-based predictors of burn-out among mothers. Fam Sc. 2014;4:148160.

40. Montgomery AJ, Peeters MCW, Schaufeli WB, Panagopoulou EP. Cross-over and work-home interference. Irish J Psych. 2008;29:61-76.

41. O'Hara MW, McCabe JE. Postpartum depression: current status and future directions. Ann Rev Clin Psych. 2013;9:379-407.

42. Olsson I, Mykletun A, Dahl AA. The Hospital Anxiety and Depression rating Scale: a cross-sectional study of psychometrics and case finding abilities in general practice. BMC Psych. 2005;5:46.

43. Paulson JF, Dauber S, Leiferman JA. Individual and combined effects of postpartum depression in mothers and fathers on parenting behavior. Ped. 2006;118:659-68.

44. Pisacane A, Continisio Gl, Aldinucci M, D'Amora S, Continisio P. A controlled trial of the father's role in breastfeeding promotion. Ped. 2005;116:494-8.

45. Pop VJ, Komproe $\mathbf{H}$, Van Son MJ. Characteristics of the Edinburgh Postnatal Depression Scale in The Netherlands. J Aff Dis. 1992;26:105-10.

46. Ramchandani P, Stein A, Evans J, O'Connor T. Paternal depression in the postnatal period. Lancet. 2005;365:2201-05.

47. Rollins J. Sharing a room: updated recommendations for a safe infant sleeping environment. Ped Nurs. 2017:43:7.

48. Rusbult CE, Martz JM, Agnew CR. The Investment Model Scale: measuring commitment level, satisfaction level, quality of alternatives, and investment size. Pers Rel. 1998:5:357-91.

49. Sanson A, Nicholson J, Ungerer J, et al. Introducing the Longitudinal Study of Australian Children (LSAC Discussion Paper No.1). Melbourne, Australia: Australian Institute of Family Studies; 2002.

50. Smeekens S, Riksen-Walraven JM, Van Bakel HJA. Multiple determinants of externalizing behavior in 5-year-olds: a longitudinal model. J Ab Child Psych. 2007;35:347-61.

51. Spinhoven PH, Ormel J, Sloekers PPA, Kempen GIJM, Speckens AEM, Van Hemert AM. A validation study of the Hospital Anxiety and Depression Scale (HADS) in different groups of Dutch subjects. Psychol Med. 1997;27:363-70.

52. Sroufe LA. Attachment and development: a prospective, longitudinal study from birth to adulthood. Attach Hum Dev. 2005;7:349-67.

53. Tabachnik BG, Fidell LS. Multilevel linear modeling. In: Hartman S, editor. Using multivariate statistics. Boston, MA: Pearson Education Inc; 2007. p. 781-857

54. Tambelli R, Cerniglia L, Cimino S, Ballarotta G. Parent-infant interactions in families with women diagnosed with postnatal depression: a longitudinal study on the effects of a psychodynamic treatment. Fron Psych. 2015;6:1210.

55. Tharner A, Luijk MCPM, Raat H, Van IJzendoorn MH, Bakermans-Kranenburg MJ, Moll HA, Jaddoe WWW, Hofman A, Verhulst FC, Tiemeier $H$. Breastfeeding and its relation to maternal sensitivity and infant attachment. J Dev Beh Ped. 2012;33:396-404

56. TNO. Peiling veilig slapen 2010/2011. Leiden: TNO; 2011.

57. TNO. Peiling melkvoeding van zuigelingen 2015. Leiden: TNO; 2015

58. Tollenaar MS, Beijers R, Jansen J, Riksen-Walraven JM, De Weerth C. Solitary sleeping in young infants is associated with heightened cortisol reactivity to a bathing session but not to a vaccination. Psychoneuroendocrinology. 2012:37:167-77.

59. Van Bussel JCH, Spitz B, Demyttenaere K. Three self-report questionnaires of the early mother-to-infant bond: reliability and validity of the Dutch version of the MPAS, PBQ and MIBS. Arch Women's Men Health. 2010;13:373-84.

60. Van Doesum KTM, Riksen-Walraven JM, Hosman CMH, Hoefnagels C. A randomized controlled trial of a home-visiting intervention aimed at 
preventing relationship problems in depressed mothers and their infants. Soc Res Child Dev. 2008;79:547-61.

61. Van Scheppingen MA, Denissen JJA, Chung JM, Tambs K, Bleidorn W. Selfesteem and relationship satisfaction during the transition to motherhood. J Pers and Soc Psych. 2017. https://doi.org/10.1037/pspp0000156.

62. Victora CG, Bahl R, Barros AJD, França GVA, Horton S, Krasevec J, Murch S, Jeeva Sankar M, Walker N, Rollins NC. Breastfeeding in the 21st century: epidemiology, mechanisms, and lifelong effect. Lancet. 2016;387:475-90

63. World Health Organization. Breastfeeding. 2017. http://www.who.int/topics/ breastfeeding/en/. Accessed 21 Mar 2017.

Submit your next manuscript to BioMed Central and we will help you at every step:

- We accept pre-submission inquiries

- Our selector tool helps you to find the most relevant journal

- We provide round the clock customer support

- Convenient online submission

- Thorough peer review

- Inclusion in PubMed and all major indexing services

- Maximum visibility for your research

Submit your manuscript at www.biomedcentral.com/submit 